\title{
ARTICLE
}

Received 17 Dec 2013 | Accepted 6 Jun 2014 | Published 10 Jul $2014 \quad$ DOl: 10.1038/ncomms5334

\section{Spin current generated by thermally driven ultrafast demagnetization}

\author{
Gyung-Min Choi ${ }^{1,2}$, Byoung-Chul Min², Kyung-Jin Lee ${ }^{3} \&$ David G. Cahill ${ }^{1}$
}

Spin current is the key element for nanoscale spintronic devices. For ultrafast operation of such nano-devices, generation of spin current in picoseconds, a timescale that is difficult to achieve using electrical circuits, is highly desired. Here we show thermally driven ultrafast demagnetization of a perpendicular ferromagnet leads to spin accumulation in a normal metal and spin transfer torque in an in-plane ferromagnet. The data are well described by models of spin generation and transport based on differences and gradients of thermodynamic parameters. The temperature difference between electrons and magnons is the driving force for spin current generation by ultrafast demagnetization. On longer timescales, a few picoseconds following laser excitation, we also observe a small contribution to spin current by a temperature gradient and the spin-dependent Seebeck effect.

\footnotetext{
${ }^{1}$ Department of Materials Science and Engineering, and Materials Research Laboratory, University of Illinois at Urbana-Champaign, 1304 West Green Street, Urbana, Illinois 61801, USA. ${ }^{2}$ Center for Spintronics Research, Korea Institute of Science and Technology, Seoul 136-791, Korea. ${ }^{3}$ Department of Materials Science and Engineering and KU-KIST Graduate School of Converging Science and Technology, Korea University, Seoul 136-701, Korea. Correspondence and requests for materials should be addressed to D.G.C. (email: d-cahill@illinois.edu).
} 
T he field of spintronics is concerned with efficient generation of spin currents and control of magnetic elements. Conventionally, spin currents are generated by passing electrical currents through ferromagnetic materials ${ }^{1-5}$. Recently, much effort has been expended to understand the generation of spin currents by thermal gradients ${ }^{6-14}$. Yet, another route to generate spin currents is rapid demagnetization of a ferromagnetic layer by direct excitation with an ultrashort laser pulse. Ultrafast demagnetization by an ultrashort laser pulse has been investigated extensively in recent years ${ }^{15-19}$, and has been described by a three-temperature model ${ }^{15}$ wherein the electronic, magnon and phonon excitations are characterized by three separate temperatures. The three-temperature model considers energy conservation, but the relaxation mechanism of spin angular momentum is controversial. Koopmans et al. ${ }^{19}$ explained rapid demagnetization by electron-phonon-mediated spin-flip scattering. Battiato et al. ${ }^{20}$ proposed that spin-dependent relaxation and fast transport of hot electrons play a crucial role and suggested that ultrafast demagnetization produces spinpolarized hot electrons, which move to an adjacent metallic layer by a so-called 'superdiffusive current'. The findings of several subsequent experiments have been interpreted as supporting this hypothesis ${ }^{21-25}$. However, the superdiffusive model is based on non-thermal electronic motion ${ }^{20}$ and the transport of thermal energy is not considered in these experiments ${ }^{21-25}$. Recently, we have shown that the exchange of thermal energy between metal layers is crucial in the interpretation of demagnetization of a ferromagnet (FM) in a metallic multilayer structure ${ }^{26}$. Therefore, we argue that a comprehensive analysis of the exchange of thermal energy in the system and the implementation of a direct method for detecting demagnetization-induced spin currents is needed.

In the following, we show that deviations from thermodynamic equilibrium can be used to describe the mechanism for spin current generation induced by demagnetization. There are three aspects to our experiments. First, we measure the demagnetization of an FM sandwiched between two normal metals (NMs) and analyse thermal transport in the sample to explain the demagnetization. Second, we measure the demagnetizationinduced spin accumulation in the NM and provide an analysis of diffusive spin transport to explain the spin accumulation. Third, we measure the demagnetization-induced spin transfer torque (STT) using an NM/FM1/NM/FM2 structure where the second magnetic layer FM2 has magnetization that is perpendicular to FM1. Previously, the generation of STT has been realized by an electric field ${ }^{1-5}$ or a temperature-gradient ${ }^{6,7}$ applied across the sample. Here we show an alternative method for generating STT using ultrafast demagnetization.

\section{Results}

Experiment. We prepared two types of ferromagnetic structures. The first type consists of, essentially, Pt $(30 \mathrm{~nm}) / \mathrm{FMl} / \mathrm{Cu}(h \mathrm{~nm})$ (we refer to this as the $\mathrm{Cu}-h \mathrm{~nm}$ sample), and the second type consists of Pt $(30 \mathrm{~nm}) / \mathrm{FM} 1 / \mathrm{Cu}(10 \mathrm{~nm}) / \mathrm{FM} 2(2 \mathrm{~nm})$ (we refer to this as the CoFeB- $2 \mathrm{~nm}$ sample). FM1 is a FM with perpendicular magnetization $\left([\mathrm{Co}(0.5 \mathrm{~nm}) / \mathrm{Pt}(1 \mathrm{~nm})]_{\times 4} / \mathrm{Co}(0.5)\right.$ or $[\mathrm{Co}$ $(0.4 \mathrm{~nm}) / \mathrm{Pt}(1 \mathrm{~nm})]_{\times 4} / \mathrm{Co}(0.2 \mathrm{~nm}) / \mathrm{Ni}(0.4 \mathrm{~nm}) / \mathrm{Co}(0.2)$ multilayer) and FM2 is an ferromagnetic CoFeB layer with in-plane magnetization (see Methods for a detailed description of the sample preparation).

The concept of how we generate a spin current from demagnetization is shown schematically in Fig. 1. The pump laser pulse excites the Pt layer and leads to the demagnetization of FM1 by thermal transport from Pt to FM1. The ultrafast demagnetization of FM1 generates a spin current by

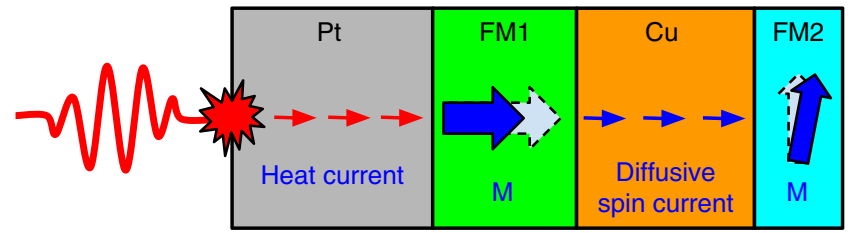

Figure 1 | Conceptual diagram. Pump laser is incident on 30-nm-thick Pt side and creates an ultrafast temperature excursion. Thermal transport from Pt to $\mathrm{FM} 1$ ( $[\mathrm{Co} / \mathrm{Pt}]$ ) drives demagnetization of the perpendicular ferromagnetic layer FM1. The demagnetization of FM1 generates a diffusive spin current in the adjacent $\mathrm{Cu}$ layer that is absorbed by the in-plane ferromagnetic layer FM2 (CoFeB). STT tilts the magnetization of FM2 out of plane and the magnetization of FM2 begins to precess.

electron-magnon coupling in FM1, as we will justify below, and by diffusive spin transport. This spin current accumulates in the $\mathrm{Cu}$ layer in the first type of structure and is absorbed by FM2 in the second type of structure. In the second type of structure, the FM1 layer has out-of-plane magnetization, whereas the FM2 layer has in-plane magnetization. This perpendicular configuration is an effective way for observing magnetization dynamics due to the spin current-induced STT; although thermal fluctuations of magnetization yield non-zero STT even for collinear magnetic configurations, the observation of STT effect in a collinear configuration requires a threshold of spin current to trigger magnetization dynamics ${ }^{4}$, while the transverse STT does not.

We apply a laser pulse on the Pt side of the samples for two reasons. The first reason is to minimize the contribution from hot electron transport with excess energy on the order of the photon energy ${ }^{20}$. The inelastic mean-free path of hot electrons in $\mathrm{Pt}$ is $\approx 5 \mathrm{~nm}^{27}$. As the $[\mathrm{Co} / \mathrm{Pt}]$ multilayer is indirectly heated by a relatively thick Pt layer $(30 \mathrm{~nm})$, the strong electron-electron and electron-phonon scattering in Pt (ref. 28) should greatly suppress the density of hot electrons that reach the $[\mathrm{Co} / \mathrm{Pt}]$ layer. When the density of far-from equilibrium hot electrons is negligible in the $[\mathrm{Co} / \mathrm{Pt}]$ layer, the superdiffusive model ${ }^{20}$ does not predict the demagnetization or a demagnetization-induced spin current. Eschenlohr et al. ${ }^{24}$ reported that $\mathrm{Ni}$ has a comparable demagnetization whether it is excited directly by laser pulse or indirectly through a Au layer. This result can be interpreted, as indicating that the superdiffusive model is applicable even when hot electrons transport through the Au layer. However, we have shown that weak electron-phonon coupling of $\mathrm{Au}$ and fast electronic transport across the $\mathrm{Au} / \mathrm{Ni}$ interface can explain the observations as well ${ }^{26}$. The second reason is to moderate the initial change of the magnetization of the CoFeB layer, which may be important to estimate spin angular momentum transfer efficiency based on the STT theory established for a single domain state $^{1,2,29}$. Substantial demagnetization of CoFeB accompanied by the simultaneous arrival of a transverse spin current may prevent us from applying the STT theory.

We use time-resolved measurements of the polar magnetooptical Kerr effect (TR-MOKE) to detect the transient magnetic and spin signals in the film-normal direction. We (i) measured the demagnetization of the $[\mathrm{Co} / \mathrm{Pt}]$ layer with the probe beam on the Pt side of the $\mathrm{Cu}-h \mathrm{~nm}$ samples; (ii) measured the spin accumulation of the $\mathrm{Cu}$ layer with the probe beam incident on the $\mathrm{Cu}$ side of the $\mathrm{Cu}-h \mathrm{~nm}$ samples; and (iii) measured the precession of the $\mathrm{CoFeB}$ layer with the probe beam on the $\mathrm{CoFeB}$ side of the CoFeB- $2 \mathrm{~nm}$ sample. In separate experiments, we use time-domain thermoreflectance (TDTR) to measure the temperature excursion of the Pt or Cu layers (see Methods for further details of the measurement techniques). 
Demagnetization of the $[\mathrm{Co} / \mathrm{Pt}]$ layer. When both pump and probe beams are incident on the Pt side of the $\mathrm{Cu}-80 \mathrm{~nm}$ sample, we observe a rapid demagnetization of the $[\mathrm{Co} / \mathrm{Pt}]$ layer on a subpicosecond timescale and partial recovery of the magnetization that is complete after a few picoseconds (Fig. 2a). The peak change in the Kerr rotation due to the rapid demagnetization is $-9.6 \pm 1 \mu \mathrm{rad}$ at $0.5 \mathrm{ps}$. We compare this value with the static Kerr rotation, $116 \pm 10 \mu \mathrm{rad}$ that we measure for the $\mathrm{Cu}-80 \mathrm{~nm}$ sample. Based on linear scaling of Kerr rotation with magnetization, we conclude that a pump fluence of $17 \mathrm{~J} \mathrm{~m}^{-2}$, which results in an absorbed fluence of $6 \mathrm{~J} \mathrm{~m}^{-2}$ (see Supplementary Note 1), creates a maximum change of magnetization of $\left.\frac{\Delta M}{M}\right|_{\max }=$ $-0.08 \pm 0.02$ at $0.5 \mathrm{ps}$ and a net change of magnetization of $\left.\frac{\Delta M}{M}\right|_{\text {net }}=-0.03 \pm 0.01$ after 3 ps.

When the $\mathrm{Cu}$ layer is sufficiently thin, the demagnetization of the $[\mathrm{Co} / \mathrm{Pt}]$ layer can also be detected by probing from the $\mathrm{Cu}$ side. With the $\mathrm{Cu}-20$ sample, we obtain $\left.\frac{\Delta M}{M}\right|_{\max }=$ $-0.07 \pm 0.02$ following the same approach as described above. When probing from the $\mathrm{Cu}$ side of the sample, the peak of the transient Kerr rotation decreases exponentially with increasing $\mathrm{Cu}$ layer thickness (see Supplementary Note 2 and Supplementary Fig. 1). This is because the Kerr rotation generated directly by the magneto-optic properties of the $[\mathrm{Co} / \mathrm{Pt}]$ layer is attenuated by the small optical transmission of thick $\mathrm{Cu}$ layers. In what follows, we focus on measurements of spin accumulation in the $\mathrm{Cu}$ layers of thickness $h>80 \mathrm{~nm}$, where the transient Kerr rotation generated directly by the change in the magnetization of the $[\mathrm{Co} / \mathrm{Pt}]$ layer is small.

We adopt the viewpoint that demagnetization is a result of excitation of magnons ${ }^{15,30,31}$ and explain the demagnetization of the $[\mathrm{Co} / \mathrm{Pt}]$ layer by the exchange of thermal energy between electrons, magnons and phonons, see Fig. $2 \mathrm{~b}$. As the $[\mathrm{Co} / \mathrm{Pt}]$ layer is indirectly heated by the Pt layer, we consider thermal energy transport from the $\mathrm{Pt}$ layer, through the $[\mathrm{Co} / \mathrm{Pt}]$ layer, and into the $\mathrm{Cu}$ layers. We measure the temperature of each layer independently: TDTR on the $\mathrm{Pt}$ side for the $\mathrm{Pt}$ phonon temperature, TDTR on the $\mathrm{Cu}$ side for the $\mathrm{Cu}$ phonon temperature and TR-MOKE on the Pt side for the $[\mathrm{Co} / \mathrm{Pt}]$ magnon temperature (see Supplementary Note 3 for details of the TDTR measurements). Next, we model the thermal transport by numerically solving coupled heat diffusion equations of $\mathrm{Pt} /[\mathrm{Co} /$ $\mathrm{Pt}] / \mathrm{Cu}$ structure that describe the transport of thermal energy by electrons and phonons in each layer (see Supplementary Note 4 for details of the thermal modelling). At $0.2 \mathrm{ps}$, the electron temperature, $T_{\mathrm{e}}$, of the $[\mathrm{Co} / \mathrm{Pt}]$ layer (blue dotted line) sharply increases by $\Delta T \approx 90 \mathrm{~K}$ due to electronic thermal transport from the Pt layer. Owing to electron-magnon coupling of $[\mathrm{Co} / \mathrm{Pt}]$, the magnon temperature, $T_{\mathrm{m}}$ (blue triangle symbol), increases by $\approx 80 \mathrm{~K}$ at $0.5 \mathrm{ps}$. (We attribute this small difference in the peak temperature of electron and magnon to the uncertainty of defining zero time delay in experiment; for the modelling, we set the zero time delay to the middle of pulse light; for the measurement, we set the zero time delay to the midpoint of the initial rise of the TDTR signal generated by Pt.) Although refs 15,30 use the term 'spin temperature' to describe the temperature of the magnon system, we prefer the term 'magnon temperature', because 'spin temperature' is sometimes used to refer to the temperature of spin-up or spin-down electrons. A temperature excursion of the magnon system of $\Delta T_{\mathrm{m}} \approx 80 \mathrm{~K}$ agrees with our observation of $\Delta M / M=-0.08$ based on the previously reported temperature-dependent magnetization of $[\mathrm{Co} / \mathrm{Pt}]^{32}$. After $1 \mathrm{ps}$, the electron temperature equilibrates with the phonon temperature, $T_{\mathrm{ph}}$, of the $[\mathrm{Co} / \mathrm{Pt}]$ layer (blue solid line), and the magnon temperature and magnetization partially recovers.

To model the spin current generation, we assume that electron-magnon coupling conserves spin angular momentum ${ }^{30}$. Thus, the spin loss by demagnetization should be converted to spin generation, that is, spin polarization in the electrons, and the spin generation rate is the negative of the demagnetization rate, $-d M / d t$ (see Supplementary Note 5). In contrast to the superdiffusive model ${ }^{20}$, our modelling is a thermal description of demagnetization-induced spin generation: the temperature difference between electron and magnon drives flows of energy and spin angular momentum. To model this effect, we include conservation of spin angular momentum in the conventional three-temperature model ${ }^{95}$.

Spin accumulation in the $\mathrm{Cu}$ layer. Figure 3 shows the transient Kerr rotation signal in the $\mathrm{Cu}$ layer, probed on the $\mathrm{Cu}$ side of the $\mathrm{Cu}-80 \mathrm{~nm}$ sample. A relatively sharp positive-going Kerr rotation is followed by a broader negative-going one (Fig. 3a,b). As the demagnetization of the $[\mathrm{Co} / \mathrm{Pt}]$ layer itself produces a negative Kerr rotation (see Fig. 2a), we conclude that the positive Kerr rotation signal is caused by spin accumulation in the $\mathrm{Cu}$ layer.
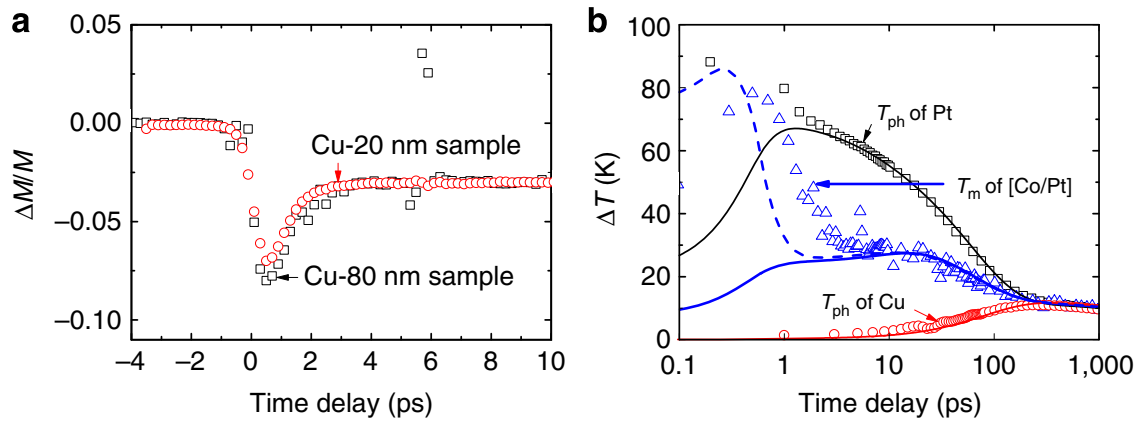

Figure 2 Demagnetization of the [Co/Pt] layer. (a) Demagnetization of the $[\mathrm{Co} / \mathrm{Pt}]$ layer of the $\mathrm{Cu}-80 \mathrm{~nm}$ sample measured with the probe beam on the Pt side (black square) and that of the $\mathrm{Cu}-20 \mathrm{~nm}$ sample with probe beam on the $\mathrm{Cu}$ side (red circle). Normalizing with static Kerr rotation, the relative change in magnetization $\triangle M / M$ is $-0.08 \pm 0.02$ and $-0.07 \pm 0.02$, respectively, at $0.5 \mathrm{ps}$ with the same pump fluence of $17.3 \mathrm{~J} \mathrm{~m}^{-2}$. The peak observed at $6 \mathrm{ps}$ is an artefact due to coherent interference between the pump and probe light due to a stray reflection. (b) The result of thermal analysis. Pt phonon temperature from a TDTR measurement on the Pt side (black square), Cu phonon temperature from a TDTR measurement on the Cu side (red circle) and [Co/Pt] magnon temperature from MOKE measurement on the Pt side (blue triangle). Solid and dotted lines are results of thermal modelling; black solid line is for Pt phonon temperatures; red solid line is for $\mathrm{Cu}$ phonon temperatures; blue solid line is for [Co/Pt] phonon temperatures; blue dotted line is for $[\mathrm{Co} / \mathrm{Pt}]$ electron temperatures. The thermal modelling is done by solving heat diffusion equations of $\mathrm{Pt} /[\mathrm{Co} / \mathrm{Pt}] / \mathrm{Cu}$ structure (see Supplementary Note 4 for details about thermal modelling). 

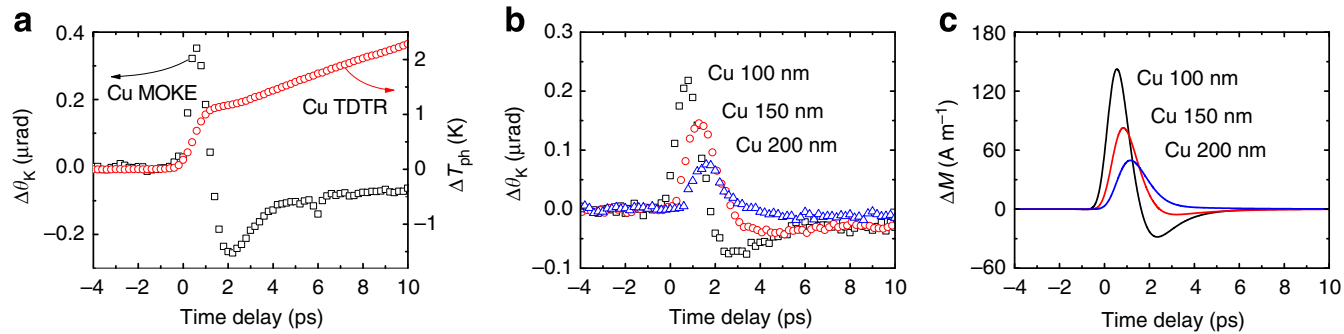

Figure 3 | Spin accumulation on the Cu layer. (a) TR-MOKE data (black square) and TDTR data (red circle) of the Cu-80 nm sample with the probe beam on the $\mathrm{Cu}$ side at pump fluence of $36 \mathrm{~J} \mathrm{~m}^{-2}$. Here we use two times larger pump fluence to increase signal-to-noise ratio. (b) Kerr rotation of $\mathrm{Cu}-100, \mathrm{Cu}-150$ and $\mathrm{Cu}-200 \mathrm{~nm}$ samples at pump fluence of $36 \mathrm{Jm}^{-2}$ (back square for $\mathrm{Cu}-100 \mathrm{~nm}$, red circle for Cu-150 nm and blue triangle for $\mathrm{Cu}-200 \mathrm{~nm}$ ). The positive Kerr rotation of the $\mathrm{Cu}-80 \mathrm{~nm}$ sample is 1.6 times larger than that of $\mathrm{Cu}-100 \mathrm{~nm}$ sample. Given the similar $\mathrm{Cu}$ thickness, we expect this increase is due to the inclusion of the $\mathrm{Co} / \mathrm{Ni}$ layer in $\mathrm{Cu}-80 \mathrm{~nm}$ sample (see Methods). (c) The spin accumulation at the end of the $\mathrm{Cu}$ layer obtained by solving coupled diffusion equation of $\mathrm{Pt}(30) /[\mathrm{Co} / \mathrm{Pt}](6.5) / \mathrm{Cu}(100 \sim 200)$ structure at pump fluence of $17 \mathrm{~J} \mathrm{~m}{ }^{-2}$; black solid line is for $\mathrm{Cu}-100 \mathrm{~nm}$; red solid line is for $\mathrm{Cu}-150 \mathrm{~nm}$; blue solid line is for $\mathrm{Cu}-200 \mathrm{~nm}$.

Figure 3a demonstrates that the timescale of the positive-going Kerr signal coincides with initial rise time of the TDTR signal. The TDTR signal is mostly controlled by $T_{\mathrm{ph}}$ of $\mathrm{Cu} ; T_{\mathrm{ph}}$ increases rapidly on short timescales because of fast energy transport by electronic excitations that are initially out of equilibrium with the $\mathrm{Cu}$ phonons. In other words, the Kerr rotation measures the nonequilibrium spin population in the $\mathrm{Cu}$ layer and the TDTR signal measures an integral of how much energy has been deposited into the phonon system by electronic thermal diffusion.

From the data plotted as Fig. 3b, we observe that the time delay corresponding to the position of the positive-going peak of Kerr rotation increases with increasing $\mathrm{Cu}$ thickness. The spin propagation velocity is on the order of $100 \mathrm{nmps}^{-1}$, much lower than Fermi velocity $\left(\sim 1,600 \mathrm{~nm} \mathrm{ps}^{-1}\right)$ of $\mathrm{Cu}$, suggesting that the spin currents we observe are mostly diffusive.

To further understand the origin of the positive and negative peaks shown in Fig. 3b, we conduct numerical simulations of timedependent spin transport. We use a diffusive model to describe spin accumulation in the $\mathrm{Cu}$ layer because we expect that ballistic hot electrons are effectively suppressed by the relatively thick Pt layer in our experiment. The spin diffusion equation is as follows ${ }^{33,34}$,

$$
\frac{\partial \mu_{\mathrm{S}}}{\partial t}=D \frac{\partial^{2} \mu_{\mathrm{S}}}{\partial z^{2}}-\frac{\mu_{\mathrm{S}}}{\tau_{\mathrm{S}}}
$$

where $\mu_{\mathrm{s}}=\mu_{\uparrow}-\mu_{\downarrow}$ is the spin chemical potential, $D$ is the spin diffusion constant and $\tau_{\mathrm{S}}$ is the spin relaxation time. The diffusion constants of $\mathrm{Pt}$ and $[\mathrm{Co} / \mathrm{Pt}]$ are 200 and $100 \mathrm{~nm}^{2} \mathrm{ps}^{-1}$, respectively, calculated by $D=\frac{\Lambda_{\mathrm{e}}}{C_{\mathrm{e}}}$, where $\Lambda_{\mathrm{e}}$ is the electronic thermal conductivity and $C_{\mathrm{e}}$ is the electronic heat capacity (see Supplementary Table 1). The spin relaxation time of $\mathrm{Pt}$ is set to $0.5 \mathrm{ps}$ using $\tau_{\mathrm{s}}=\frac{l_{\mathrm{s}}^{2}}{D}$, where $l_{\mathrm{S}}$ is the spin diffusion length; $l_{\mathrm{S}}=10 \mathrm{~nm}$ in $\mathrm{Pt}$ (ref. 35). For $\mathrm{Cu}, D=6,500 \mathrm{~nm}^{2} \mathrm{ps}^{-1}$ and $\tau_{\mathrm{S}}=25 \mathrm{ps}$ (ref. 36). The $\tau_{\mathrm{S}}$ of [Co/Pt] has not been reported; hence, we use as an estimate $\tau_{\mathrm{s}}=\frac{\tau_{0}}{a_{\mathrm{sf}}}$, where $\tau_{0}$ is the momentum relaxation time and $a_{\mathrm{sf}}$ is the spin-flip probability from theory of Elliot-Yafet ${ }^{37,38}$. The $\tau_{0}$ of $[\mathrm{Co} / \mathrm{Pt}]$ is calculated to be $5 \mathrm{fs}$ using $\tau_{0}=\frac{3 D}{v_{\mathrm{F}}^{2}}$, where $v_{\mathrm{F}}$ is the Fermi velocity; $v_{\mathrm{F}}=0.24 \times 10^{6} \mathrm{~m} \mathrm{~s}^{-1}$ for Pt [111] direction ${ }^{39}$. The $a_{\text {sf }}$ of typical ferromagnetic transition metals is $0.1 \sim 0.2$ (ref. 19) and that of Co has been calculated to be $0.01 \sim 0.02$ (ref. 40). Considering multiple interfaces of the $[\mathrm{Co} / \mathrm{Pt}]$ layer, we use $a_{\mathrm{sf}}=0.1$ and $\tau_{0}=5 \mathrm{fs}$; the $\tau_{\mathrm{S}}$ of $[\mathrm{Co} / \mathrm{Pt}]$ is then $0.05 \mathrm{ps}$. Although $\tau_{\mathrm{S}}$ determined in this way has large uncertainties, we find $\tau_{\mathrm{S}}=0.05 \mathrm{ps}$ of $[\mathrm{Co} / \mathrm{Pt}]$ agrees with the STT result described below.

We solve the coupled diffusion equations for $\mathrm{Pt}(30) /[\mathrm{Co} / \mathrm{Pt}]$ $(6.5) / \mathrm{Cu}(100 \sim 200)$ structures by equating the spin chemical potential at the interfaces. (We do not include a finite spin conductance in this modelling, which we will use later in analysing the STT experiment, because we found that a finite spin conductance does not significantly change the predicted spin accumulation in these relatively thick $\mathrm{Cu}$ layers.) We include the spin generation term in the $[\mathrm{Co} / \mathrm{Pt}]$ layer by assuming that the spin generation rate is $-d M / d t ; d M / d t$ is obtained by numerical differentiation of the demagnetization data shown in Fig. 2a (see Supplementary Fig. 2).

The predicted spin accumulation at the end of the $\mathrm{Cu}$ layer with different $\mathrm{Cu}$ thickness is shown in Fig. 3c. The overall timedependent behaviour is in excellent agreement with that of the transient Kerr rotation (Fig. 3b). For example, the broad negative peak following the positive peak is reproduced by our model of spin diffusion, implying that diffusive spin transport and spin generation by ultrafast demagnetization captures the key features of the time-dependent spin accumulation in the $\mathrm{Cu}$ layer. This result supports our assumption that we can describe spin generation and spin transport in our samples in terms of differences and gradients in thermodynamic parameters.

Spin current by a temperature gradient. However, we also observe an important difference between the model and the experiment at delay times beyond a few picoseconds. The measured Kerr signal does not decay to zero at $10 \mathrm{ps,} \mathrm{whereas} \mathrm{the}$ calculated spin accumulation does decay to zero. We attribute this small signal at long delay times to a spin current generated by the temperature gradient of the $[\mathrm{Co} / \mathrm{Pt}]$ layer. The ultrashort light pulse produces not only a temperature difference between different heat reservoirs (electrons, magnons and phonons) but also differences in temperatures at different locations in the sample. Slachter et al. ${ }^{8}$ showed that a steady temperature gradient in $\mathrm{NiFe}$ produces spin current at the $\mathrm{NiFe} / \mathrm{Cu}$ interface due to the spindependent Seebeck effect. Following the analysis of ref. 8, the spin current, $J_{\mathrm{S}}$, is related to the the spatial temperature gradient by,

$$
J_{\mathrm{S}}=-\frac{\mu_{\mathrm{B}}}{e}\left(\sigma_{\uparrow} S_{\uparrow}-\sigma_{\downarrow} S_{\downarrow}\right) \nabla T=-\frac{\mu_{\mathrm{B}}}{e} \frac{\sigma_{\uparrow} S_{\uparrow}-\sigma_{\downarrow} S_{\downarrow}}{\sigma_{\uparrow}+\sigma_{\downarrow}} \sigma \nabla T
$$

where $\mu_{\mathrm{B}}$ is the Bohr magneton, $e$ is the elementary charge, $\sigma_{\uparrow, \downarrow}$ is the spin-dependent electric conductivity, $S_{\uparrow, \downarrow}$ is the spindependent Seebeck coefficient and $\nabla T$ is the electronic temperature gradient. The spin-dependent Seebeck coefficient leads to an abrupt change of spin current going from FM to NM and thereby generates spin current at the $\mathrm{Pt} /[\mathrm{Co} / \mathrm{Pt}]$ and $[\mathrm{Co} / \mathrm{Pt}] /$ $\mathrm{Cu}$ interfaces with opposite $\operatorname{sign}^{8}$. 

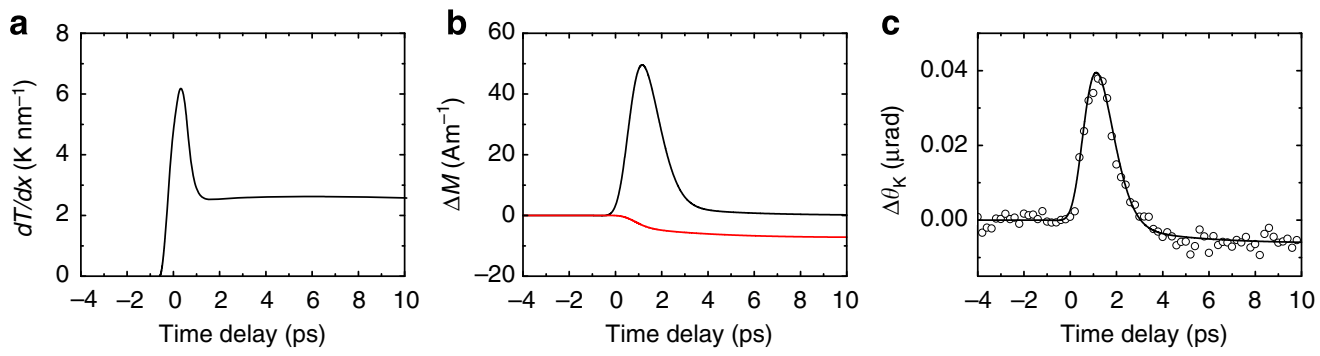

Figure 4 | Spin current by spatial temperature gradient. (a) Estimation of the electronic temperature gradient of [Co/Pt] in the Cu-200 nm sample from the thermal analysis. (b) Estimation of the spin accumulation at the end of Cu layer of Cu-200 nm sample with the $\nabla T$-driven spin current (red solid line). For comparison, the black solid line is the spin accumulation due to the demagnetization-induced spin current in Fig. 3c. All calculations are done with a pump fluence of $17 \mathrm{Jm}^{-2}$. (c) Comparison between the spin accumulation data (black circle) and the simulated result (black solid line) of the Cu-200 nm sample with a pump fluence of of $17 \mathrm{~J} \mathrm{~m}^{-2}$. The spin accumulation data is taken from Fig. $3 \mathrm{~b}$ and divided by a factor of two because the data is obtained with a pump fluence of $35 \mathrm{Jm}^{-2}$. The time delay of the data has been shifted to $-0.4 \mathrm{ps}$ for matching with the simulation. The simulation result (black solid line) is the sum of black solid line and red solid line of $\mathbf{b}$.

We analyse the $\mathrm{Cu}-200 \mathrm{~nm}$ sample to make sure that the demagnetization signal of $[\mathrm{Co} / \mathrm{Pt}]$ is completely suppressed by a thick $\mathrm{Cu}$ layer (see Supplementary Note 2). In Fig. 4a, we estimate the time dependence of the temperature gradient $\nabla T(t)$ of the $[\mathrm{Co} / \mathrm{Pt}]$ layer from the analysis of thermal transport in the sample described above. With this $\nabla T$ and $\sigma=2.7 \times 10^{6} \Omega^{-1} \mathrm{~m}^{-1}$, which is obtained by applying Wiedemann-Franz law to the thermal conductivity of the $[\mathrm{Co} / \mathrm{Pt}]$ layer, we calculate the $\nabla T$ driven spin accumulation of the $\mathrm{Cu}-200 \mathrm{~nm}$ sample using our spin diffusion modelling. By comparing the experimental Kerr rotation and the predicted spin accumulation, see Fig. $4 \mathrm{~b}, \mathrm{c}$, we obtain a best fit to the data using $\frac{\sigma_{\uparrow} S_{\uparrow}-\sigma_{\downarrow} S_{\downarrow}}{\sigma_{\uparrow}+\sigma_{\downarrow}} \approx 5 \mu \mathrm{VK}^{-1}$.

We also determine the relationship between Kerr rotation and spin accumulation in $\mathrm{Cu}$ by comparing the experiment with the model: we find $\left.\frac{\Delta \theta_{\mathrm{K}}}{\Delta M}\right|_{\mathrm{cu}} \approx 10^{-9} \mathrm{rad} \mathrm{m} \mathrm{A}{ }^{-1}$. Kerr rotation in NMs in an external magnetic field has been reported previously ${ }^{41,42}$; a magnetic field produces spin accumulation in a NM by splitting energy of spin sub-bands. However, Kerr rotation in an applied field has an additional contribution from field-dependent scattering. As we measure the spin accumulation in $\mathrm{Cu}$ without an applied magnetic field, the field-dependent scattering term is absent and our measurement is only sensitive to spin accumulation.

STT in the CoFeB layer. The demagnetization-induced spin current discussed above has a spin component collinear with the $[\mathrm{Co} / \mathrm{Pt}]$ magnetization. For the CoFeB- $2 \mathrm{~nm}$ sample, therefore, this spin current is transverse to the $\mathrm{CoFeB}$ magnetization and could efficiently supply STT. The CoFeB layer acts as a spin sink due to a rapid dephasing of the transverse spin component within a few atomic layers of a $\mathrm{FM}^{29}$. The absorption of the transverse spin current by the $\mathrm{CoFeB}$ layer produces a tilting of its magnetization followed by precession.

The most salient feature observed with the CoFeB- $2 \mathrm{~nm}$ sample is that the precession of the $\mathrm{CoFeB}$ layer appears on top of the smooth background created by the demagnetization and magnetization recovery of the $[\mathrm{Co} / \mathrm{Pt}]$ layer (Fig. $5 \mathrm{a}$ ). (The $\mathrm{Cu}$ layer in this sample is thin, only $10 \mathrm{~nm}$, and therefore the magneto-optic properties of the $[\mathrm{Co} / \mathrm{Pt}]$ directly effects the polarization of the probe.) Spin current from the $[\mathrm{Co} / \mathrm{Pt}]$ layer tilts the $\mathrm{CoFeB}$-magnetization - which initially lies in-plane-outof-plane within a few picoseconds; the $\mathrm{CoFeB}$ magnetization subsequently precesses. The initial tilting of $\mathrm{CoFeB}$ produces a positive Kerr rotation, while the demagnetization of $[\mathrm{Co} / \mathrm{Pt}]$ produces a negative rotation. As the static Kerr rotations of $\mathrm{CoFeB}$ and $[\mathrm{Co} / \mathrm{Pt}]$ are of the same sign, the direction of the initial $\mathrm{CoFeB}$ tilting is the same as that of the $[\mathrm{Co} / \mathrm{Pt}]$ magnetization. The demagnetization-induced spin current should tilt the $\mathrm{CoFeB}$ magnetization to the direction of the $\mathrm{Co} / \mathrm{Pt}$ magnetization, but the temperature gradient-induced spin current should tilt the CoFeB magnetization to the opposite direction (see Fig. 4b). Therefore, we conclude that the demagnetization is more dominant than the temperature gradient. We discuss other possible origins for precession of the $\mathrm{CoFeB}$ magnetization and the possibility of $[\mathrm{Co} / \mathrm{Pt}]$-precession in Supplementary Note 6 and Supplementary Fig. 3.

After subtracting the demagnetization signal, the precession signal is well described by a damped sine function whose frequency, $7.4 \mathrm{GHz}$, and damping constant, 0.018 , are can be calculated from the properties of $\mathrm{CoFeB}$ (see Supplementary Note 7 and Supplementary Fig. 4). The oscillation amplitude is $0.45 \pm 0.5 \mu \mathrm{rad}$ at a pump fluence of $17 \mathrm{~J} \mathrm{~m}^{-2}$. Comparing with the static Kerr rotation, $240 \pm 50 \mu \mathrm{rad}$, of the full magnetization of the $\mathrm{CoFeB}$ layer, the oscillation amplitude corresponds to $\frac{\Delta M}{M}=2 \pm 0.6 \times 10^{-3}$.

The precession amplitude of $\mathrm{CoFeB}$ is linearly proportional to the pump fluence and approach zero at zero pump fluence (Fig. 5b). This result is characteristic of the transverse STT. For collinear STT, where two magnetizations lie parallel or antiparallel, there should be a threshold of spin current to trigger a precession ${ }^{4}$. However, for the transverse STT, there should be no threshold, and the precession amplitude should go smoothly to zero at zero spin current, in agreement with our observations. We also observe that the precession amplitude of $\mathrm{CoFeB}$ decreases as the angle between $[\mathrm{Co} / \mathrm{Pt}]$ and $\mathrm{CoFeB}$ deviates from transverse configuration (see Supplementary Note 8 and Supplementary Fig. 5).

We compare the spin gain of the CoFeB layer and the spin loss of the $[\mathrm{Co} / \mathrm{Pt}]$ layer. For the $\mathrm{CoFeB}$ layer, we calculate the spin gain as $4.8 \times 10^{-6} \mathrm{~A}$ by multiplying $M_{\mathrm{S}}\left(=1.2 \times 10^{6} \mathrm{~A} \mathrm{~m}^{-1}\right)$, thickness $\left(=2 \times 10^{-9} \mathrm{~m}\right)$ and $\frac{\Delta M}{M}(=0.002)$. For the $[\mathrm{Co} / \mathrm{Pt}]$ layer, the spin loss by demagnetization is $7.8 \times 10^{-5} \mathrm{~A}$, calculated in the same manner by multiplying $M_{\mathrm{S}}\left(=4 \times 10^{5} \mathrm{Am}^{-1}\right)$, thickness $\left(=6.5 \times 10^{-9} \mathrm{~m}\right)$ and $\left.\frac{\Delta M}{M}\right|_{\text {net }}(=0.03)$. Thus, $6 \%$ of the spin loss of the $[\mathrm{Co} / \mathrm{Pt}]$ layer is converted to the spin gain of the $\mathrm{CoFeB}$ layer.

For a more quantitative estimate of the spin transfer, we solve the spin diffusion equation for the four-layer $\mathrm{Pt} /[\mathrm{Co} / \mathrm{Pt}] / \mathrm{Cu} /$ $\mathrm{CoFeB}$ structure with the diffusion constant and relaxation time of each layer as described above. We set $\mu_{\mathrm{s}}=0$ in the CoFeB layer as the $\mathrm{CoFeB}$ layer acts as a spin sink. We also include finite spin conductances at NM-FM interfaces ${ }^{43,44}$, because the interfacial spin conductance becomes dominant over the bulk diffusivity at 

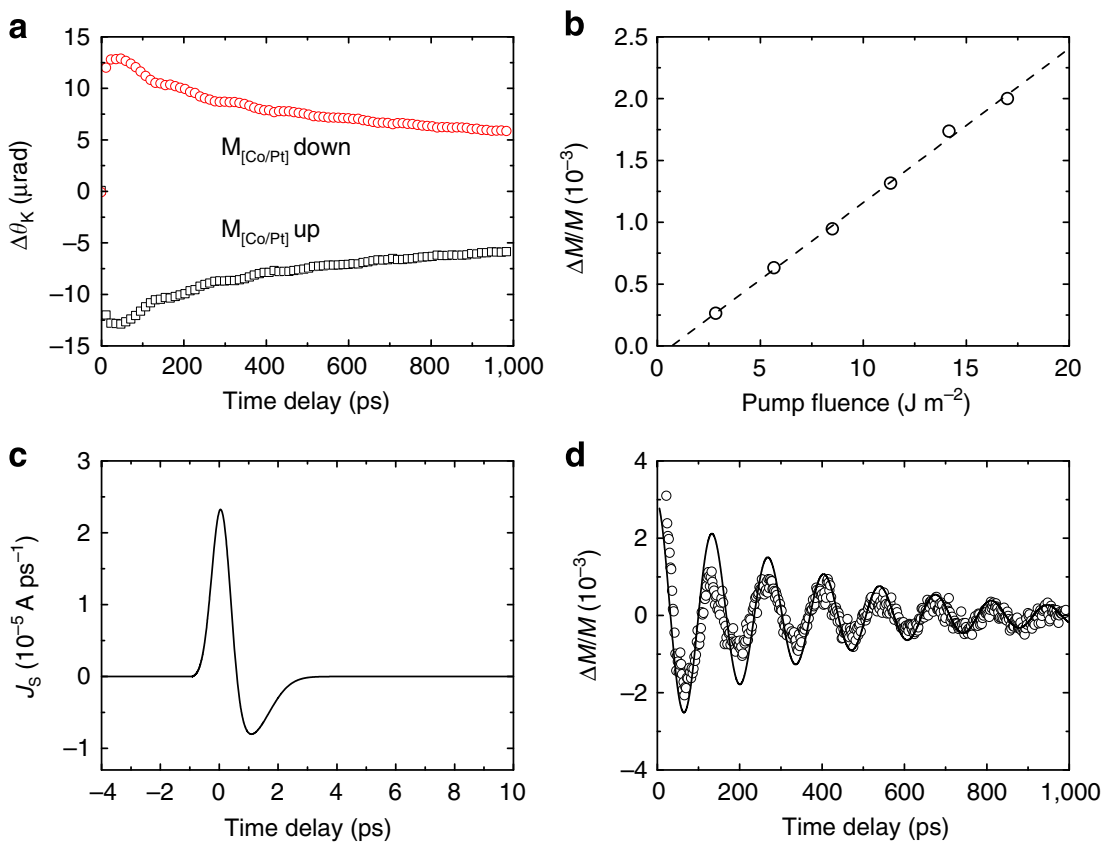

Figure 5 I STT on the CoFeB layer. (a) Kerr rotation of the CoFeB- $2 \mathrm{~nm}$ sample with pump fluence of $17 \mathrm{~J} \mathrm{~m}{ }^{-2}$ with the [Co/Pt] magnetization up (black square) or down (red circle). (b) The normalized precession amplitude, $\Delta M / M$, of the CoFeB with different pump fluence up to $17 \mathrm{~J} \mathrm{~m}^{-2}$ (black circle). The dotted line is the linear fitting of data. (c) The calculated spin current that goes to CoFeB in the CoFeB- $2 \mathrm{~nm}$ sample. The calculation is done by the spin diffusion simulation. (d) The precession data and magnetization dynamics simulation. The precession data (black circle) is obtained by subtracting the demagnetization signal of $\mathbf{a}$ at the $[\mathrm{Co} / \mathrm{Pt}]$ magnetization up. The magnetization dynamics simulation (black solid line) is done by Landau-Lifshitz-Gilbert equation with an input spin current of $\mathbf{c}$.

thin $\mathrm{Cu}$ thickness. For the longitudinal component, the spin conductance is $\frac{G_{\uparrow}+G_{\downarrow}}{2 e^{2}}$, and for the transverse component the spin conductance is $\frac{\operatorname{Re}\left\{G_{\downarrow \downarrow}\right\}}{e^{2}}$, where $G_{\uparrow}$ is the conductance of the majority spin, $G_{\downarrow}$ is that of the minority spin and $G_{\uparrow \downarrow}$ is the spin mixing conductance ${ }^{44}$. As the spin in $\mathrm{Cu}$ and $\mathrm{Pt}$ is collinear with the $[\mathrm{Co} / \mathrm{Pt}]$ magnetization and transverse with the $\mathrm{CoFeB}$ magnetization, we incorporate the longitudinal spin conductance in the model at the $\mathrm{Pt} /[\mathrm{Co} / \mathrm{Pt}]$ and $[\mathrm{Co} / \mathrm{Pt}] / \mathrm{Cu}$ interfaces, and the transverse spin conductance at the $\mathrm{Cu} / \mathrm{CoFeB}$ interface. We use values for the spin conductances from the first-principles calculation of the $\mathrm{Co} / \mathrm{Cu}$ interface ${ }^{45}, G_{\uparrow}=0.42, G_{\downarrow}=0.33$ and $\operatorname{Re}\left\{G_{\uparrow \downarrow}\right\}=0.56 \times 10^{15} \Omega^{-1} \mathrm{~m}^{-2}$. Performing spin diffusion simulation with these parameters, we determine the spin current that goes to $\mathrm{CoFeB}$ (Fig. 5c). Owing to the small spin relaxation time of $[\mathrm{Co} / \mathrm{Pt}]$ and finite spin conductances at interfaces, only $9 \%$ of total spin generation by $d M / d t$ of $[\mathrm{Co} / \mathrm{Pt}]$ goes to $\mathrm{CoFeB}$.

Using the obtained $J_{S}$ as an input, we perform magnetization dynamics simulation for CoFeB with the Landau-Lifshitz-Gilbert equation incorporating $\mathrm{STT}^{46-48}$,

$$
\dot{\mathbf{m}}=-\gamma \mathbf{m} \times \mathbf{H}_{\text {eff }}+\alpha \mathbf{m} \times \dot{\mathbf{m}}+\frac{J_{\mathrm{S}}}{M_{\mathrm{S}} h} \mathbf{m} \times\left(\mathbf{m} \times \mathbf{m}_{\text {fixed }}\right)
$$

where $\mathbf{m}$ and $\mathbf{m}_{\text {fixed }}$ are unit vectors in the direction of the CoFeB and $[\mathrm{Co} / \mathrm{Pt}]$ magnetizations, respectively, $\dot{\mathbf{m}}$ is the time derivative of $\mathbf{m}, M_{\mathrm{S}}$ and $h$ are the saturation magnetization and thickness of the $\mathrm{CoFeB}$ layer, respectively, $\mathbf{H}_{\text {eff }}$ is the effective field due to applied field and shape anisotropy of $\mathrm{CoFeB}, \gamma$ is the gyromagnetic ratio, $\alpha$ is the Gilbert damping constant and $J_{S}$ is the spin current. We use the same values for applied field, shape anisotropy and $\alpha$ of $\mathrm{CoFeB}$ as in Supplementary Note 7. The simulation result is in good agreement with the precession data (Fig. 5d). The precession amplitude of the simulation is higher than that of the measurement, but considering uncertainties in the spin relaxation times, interfacial spin conductances and measurements, the agreement between data and simulation is good.

\section{Discussion}

Our models explain the experimentally observed spin current generation within the context of temperature differences between thermal reservoirs and temperature differences between different locations in the sample, that is, we successfully describe the spin current generation in terms of deviations from thermodynamic equilibrium. Electron-magnon coupling and differences in the temperatures of electrons and magnons in a rapidly heated ferromagnetic layer converts demagnetization to spin generation in the FM. The generated spin leads to spin accumulation in an adjacent NM and STT in a second ferromagnetic layer by diffusive spin transport. The demagnetization-induced STT occurs on picosecond timescales that are extremely challenging to achieve in electrical measurements. Thus, we refer to this spin current as thermally driven and believe that our results extend the emerging discipline of spin caloritronics into the regime of picosecond timescales. Our study provides new insights on the physical mechanisms governing the interplay of spin and heat at the nanoscale and addresses the fundamental limits of ultrafast spintronic devices for data storage and information processing.

\section{Methods}

Sample preparation and characterization. To observe spin accumulation in $\mathrm{Cu}$ driven by laser heating, we use a film structure of sapphire substrate/Pt (30)/[Co $(0.4) / \mathrm{Pt}(1)]_{\times 4} / \mathrm{Co}(0.2) / \mathrm{Ni}(0.4) / \mathrm{Co}(0.2) / \mathrm{Cu}(80) / \mathrm{MgO}(10) / \mathrm{Al}_{2} \mathrm{O}_{3}$ (5), where the numerical values in parenthesis are the layer thickness in nanometres. This sample is prepared using a seven-target sputter deposition system with a base pressure of $<5 \times 10^{-8}$ Torr at the Korea Institute of Science and Technology. The $[\mathrm{Co} / \mathrm{Pt}]$ layer is a metallic FM with perpendicular magnetic anisotropy. We characterized the magnetic properties of the $[\mathrm{Co} / \mathrm{Pt}]$ layer with a vibrating sample magnetometer; 
the saturation magnetization is $4 \times 10^{5} \mathrm{~A} \mathrm{~m}^{-1}$, the coercivity is $0.1 \mathrm{~T}$ and the remanent magnetization is approximately the same as the saturation magnetization. We insert a thin $\mathrm{Co} / \mathrm{Ni}$ layer between $[\mathrm{Co} / \mathrm{Pt}]$ and $\mathrm{Cu}$ layers to minimize spin scattering by the $\mathrm{Pt}$ layer ${ }^{5}$. We chose a Cu layer as the spin accumulation layer because of its relatively long spin diffusion length ${ }^{36} . \mathrm{MgO}$ and $\mathrm{Al}_{2} \mathrm{O}_{3}$ layers are deposited to protect the $\mathrm{Cu}$ layer from oxidation. The $\mathrm{MgO}$ layer is sputtered deposited in the same deposition chamber as the metal layers; the $\mathrm{Al}_{2} \mathrm{O}_{3}$ layer is deposited in a separate deposition system.

To observe STT, we use a sample incorporating a second magnetic layer with inplane magnetization. This sample has the structure sapphire substrate/Pt (30)/[Co $(0.4) / \mathrm{Pt}(1)]_{\times 4} / \mathrm{Co}(0.2) / \mathrm{Ni}(0.4) / \mathrm{Co}(0.2) / \mathrm{Cu}(10) / \mathrm{CoFeB}(2) / \mathrm{MgO}(10) / \mathrm{Al}_{2} \mathrm{O}_{3}$ (5), prepared at the Korea Institute of Science and Technology. The saturation magnetization of the CoFeB layer of $1.4 \times 10^{6} \mathrm{~A} \mathrm{~m}^{-1}$ is determined with thermally oxidized Si substrate/CoFeB (45) sample. To fit the precession result of $\mathrm{CoFeB}-2 \mathrm{~nm}$ sample with damped sine function, we reduced $M_{\mathrm{S}}$ of $\mathrm{CoFeB}$ to $1.2 \times 10^{6} \mathrm{~A} \mathrm{~m}^{-1}$. We expect this reduction is due to thin thickness of the CoFeB layer.

We prepared an additional set of samples with varying $\mathrm{Cu}$ thickness between 10 and $200 \mathrm{~nm}$ using a three-target sputter deposition system at the University of Illinois. The film structure is sapphire substrate/Pt (30)/[Co (0.5)/ $\mathrm{Pt}(1)]_{\times 4} / \mathrm{Co}$ $(0.5) / \mathrm{Cu}(h) / \mathrm{SiO}_{2}(10)$, where $h$ denotes the $\mathrm{Cu}$ thickness $10<h<200 \mathrm{~nm}$. We could not include the Ni layer in these structures because of the limitation on the number of targets. The $\mathrm{SiO}_{2}$ layer is prepared by e-beam evaporator in a separate deposition system.

Pump-probe measurement. The wavelengths of the pump and probe pulses are centred at $785 \mathrm{~nm}$ and have a FWHM) spectral range of $\sim 10 \mathrm{~nm}$. The $1 / e^{2}$ intensity radius of the focused pump and probe are $5 \mu \mathrm{m}$. Pump optical pulses are incident on the Pt side of the samples through the sapphire substrate. As the pump pulses pass through an electro-optic modulator with a relatively large group-delaydispersion, the FWHM duration of the pump pulses is relatively long, $\sim 0.8 \mathrm{ps}$. The FWHM duration of the probe pulses is $\sim 0.35 \mathrm{ps}$.

We use TR-MOKE to detect the transient magnetic properties. The incident probe is linearly polarized; the rotation of the polarization on reflection from the surface of the sample is measured by splitting the probe beam with a Wollaston prism and detecting the changes in the intensity of the orthogonal polarization states using a balanced detector. The changes in the light intensity convert to the voltage signal after the balanced detector. We obtain the Kerr rotation from the voltage signal using a measured conversion factor between the voltage and rotation. We use a double modulation technique with the pump beam modulated at $10.7 \mathrm{MHz}$ and the probe beam modulated at $200 \mathrm{~Hz}$ to further improve the signalto-noise ratio and suppress background created by a diffusely scattered pump light. We set the zero time delay to the midpoint of the initial rise of the TDTR signal generated by Pt. Except for the precession measurement where we apply an external magnetic field of $0.045 \mathrm{~T}$ in the in-plane direction, all the measurements are conducted without an applied magnetic field.

\section{References}

1. Slonczewski, J. C. Current-driven excitation of magnetic multilayers. J. Magn. Magn. Mater. 159, L1-L7 (1996).

2. Berger, L. Emission of spin waves by a magnetic multilayer traversed by a current. Phys. Rev. B 54, 9353-9358 (1996).

3. Tsoi, M. et al. Excitations of a magnetic multilayer by an electric current. Phys. Rev. Lett. 80, 4281-4284 (1998).

4. Katine, J. A., Albert, F. J., Buhrman, R. A., Myers, E. B. \& Ralph, D. C. Currentdriven magnetization reversal and spin-wave excitations in $\mathrm{Co} / \mathrm{Cu} / \mathrm{Co}$ pillars. Phys. Rev. Lett. 84, 3149-3152 (2000).

5. Mangin, S. et al. Current-induced magnetization reversal in nanopillars with perpendicular anisotropy. Nat. Mater. 5, 210-215 (2006).

6. Hatami, M., Bauer, G. E. W., Zhang, Q. \& Kelly, P. J. Thermal spin-transfer torque in magnetoelectronic devices. Phys. Rev. Lett. 99, 066603 (2007).

7. Yu, H., Granville, S., Yu, D. P. \& Ansermet, J.-P. Evidence for thermal spin-transfer torque. Phys. Rev. Lett. 104, 146601 (2010).

8. Slachter, A., Bakker, F. L., Adam, J.-P. \& van Wees, B. J. Thermally driven spin injection from a ferromagnet into a non-magnetic metal. Nat. Phys. 6, 879-882 (2010).

9. Slonczewski, J. C. Initiation of spin-transfer torque by thermal transport from magnons. Phys. Rev. B 82, 054403 (2010).

10. Uchida, K. et al. Spin Seebeck insulator. Nat. Mater. 9, 894-897 (2010).

11. Huang, S. Y., Wang, W. G., Lee, S. F., Kwo, J. \& Chien, C. L. Intrinsic spindependent thermal transport. Phys. Rev. Lett. 107, 216604 (2011).

12. Krause, S., Herzog, G., Schlenhoff, A., Sonntag, A. \& Wiesendanger, R. Joule heating and spin-transfer torque investigated on the atomic scale using a spinpolarized scanning tunneling microscope. Phys. Rev. Lett. 107, 186601 (2011).

13. Padrón-Hernández, E., Azevedo, A. \& Rezende, S. M. Amplification of spin waves by thermal spin-transfer torque. Phys. Rev. Lett. 107, 197203 (2011).

14. Brechet, S. D., Vetro, F. A., Papa, E., Barnes, S. E. \& Ansermet, J.-P. Evidence for a magnetic Seebeck effect. Phys. Rev. Lett. 111, 087205 (2013)

15. Beaurepaire, E., Merle, J.-C., Daunois, A. \& Bigot, J.-Y. Ultrafast spin dynamics in ferromagnetic nickel. Phys. Rev. Lett. 76, 4250-4253 (1996).
16. Koopmans, B., Ruigrok, J. J. M., Longa, F. D. \& de Jonge, W. J. M. Unifying ultrafast magnetization dynamics. Phys. Rev. Lett. 95, 267207 (2005).

17. Stamm, C. et al. Femtosecond modification of electron localization and transfer of angular momentum in nickel. Nat. Mater. 6, 740-743 (2007).

18. Boeglin, C. et al. Distinguishing the ultrafast dynamics of spin and orbital moments in solids. Nature 465, 458-461 (2010).

19. Koopmans, B. et al. Explaining the paradoxical diversity of ultrafast laserinduced demagnetization. Nat. Mater. 9, 259-265 (2010).

20. Battiato, M., Carva, K. \& Oppeneer, P. M. Superdiffusive spin transport as a mechanism of ultrafast demagnetization. Phys. Rev. Lett. 105, 027203 (2010).

21. Malinowski, G. et al. Control of speed and efficiency of ultrafast demagnetization by direct transfer of spin angular momentum. Nat. Phys. 4, 855-858 (2008).

22. Melnikov, A. et al. Ultrafast transport of laser-excited spin-polarized carriers in $\mathrm{Au} / \mathrm{Fe} / \mathrm{MgO}(001)$. Phys. Rev. Lett. 107, 076601 (2011).

23. Rudolf, D. et al. Ultrafast magnetization enhancement in metallic multilayers driven by superdiffusive spin current. Nat. Commun. 3, 1037 (2012).

24. Eschenlohr, A. et al. Ultrafast spin transport as key to femtosecond demagnetization. Nat. Mater. 12, 332-336 (2013).

25. Turgut, E. et al. Controlling the competition between optically induced ultrafast spin-flip scattering and spin transport in magnetic multilayers. Phys. Rev. Lett. 110, 197201 (2013).

26. Choi, G.-M., Wilson, R. B. \& Cahill, D. G. Indirect heating of Pt by short-pulse laser irradiation of $\mathrm{Au}$ in a nanoscale Pt/Au bilayer. Phys. Rev. B 89, 064307 (2014).

27. Chulkov, E. V. et al. Electronic excitations in metals and at metal surfaces. Chem. Rev. 106, 4160-4206 (2006).

28. Lin, Z. \& Zhigilei, L. V. Electron-phonon coupling and electron heat capacity of metals under conditions of strong electron-phonon nonequilibrium. Phys. Rev B 77, 075133 (2008).

29. Stiles, M. D. \& Zangwill, A. Anatomy of spin-transfer torque. Phys. Rev. B 66, 014407 (2002)

30. Manchon, A., Li, Q., Xu, L. \& Zhang, S. Theory of laser-induced demagnetization at high temperature. Phys. Rev. B 85, 064408 (2012).

31. Schellekens, A. J. \& Koopmans, B. Comparing ultrafast demagnetization rates between competing models for finite temperature magnetism. Phys. Rev. Lett. 110, 217204 (2013).

32. McDaniel, T. W. \& Victora, R. H. (eds) Handbook of Magneto-Optical Data Recording: Materials, Subsystems, Techniques (Noyes Publications, 1995).

33. Valet, T. \& Fert, A. Theory of the perpendicular magnetoresistance in magnetic multilayers. Phys. Rev. B 48, 7099-7113 (1993).

34. Stiles, M. D., Xiao, J. \& Zangwill, A. Phenomenological theory of currentinduced magnetization precession. Phys. Rev. B 69, 054408 (2004).

35. Niimi, Y. et al. Experimental verification of comparability between spin-orbit and spin-diffusion length. Phys. Rev. Lett. 110, 016805 (2013).

36. McKenna, K. P. \& Morgan, G. J. Quantum simulations of spin-relaxation and transport in copper. Eur. Phys. J. B 59, 451-456 (2007).

37. Elliot, R. J. Theory of the effect of spin-orbit coupling on magnetic resonance in some semiconductors. Phys. Rev. 96, 266-279 (1954).

38. Yafet, Y. Solid State Physics. (eds Seitz, F. \& Turnbull, D.) Vol. 14 (Academic Press, 1963).

39. Dye, D. H., Ketterson, J. B. \& Crabtree, G. W. The Fermi surface of platinum. J. Low Temp. Phys. 30, 813-838 (1978).

40. Carva, K., Battiato, M., Legut, D. \& Oppeneer, P. M. Ab initio theory of electron-phonon mediated ultrafast spin relaxation of laser-excited hot electrons in transition-metal ferromagnets. Phys. Rev. B 87, 184425 (2013).

41. Schnatterly, S. E. Magnetoreflection measurement on the noble metals. Phys. Rev. 183, 664-667 (1969).

42. Elezzabi, A. Y. \& Freeman, M. R. Direct measurement of the conduction electron spin-lattice relaxation time $T_{1}$ in gold. Phys. Rev. Lett. 77, 3220-3223 (1996).

43. Brataas, A., Nazarov, Y. N. \& Bauer, G. E. W. Spin-transport in multi-terminal normal metal-ferromagnet systems with non-collinear magnetizations. Eur. Phys. J. B 22, 99-110 (2001).

44. Barnaś, J., Fert, A., Gmitra, M., Weymann, I. \& Dugaev, V. K. From giant magnetoresistance to current-induced switching by spin transfer. Phys. Rev. B 72, 024426 (2005).

45. Zwierzycki, M., Tserkovnyak, Y., Kelly, P. J., Brataas, A. \& Bauer, G. E. W. First-principles study of magnetization relaxation enhancement and spin transfer in thin magnetic films. Phys. Rev. B 71, 064420 (2005).

46. Landau, L. \& Lifshitz, E. Theory of the dispersion of magnetic permeability in ferromagnetic bodies. Phys. Z. Sowietunion 8, 153-169 (1935).

47. Gilbert, T. L. IEEE Trans. Magn. 40, 3443-3449 (2004).

48. Slonczewski, J. C. Currents and torques in metallic magnetic multilayers. J. Magn. Magn. Mater. 247, 324-338 (2002).

\section{Acknowledgements}

The development of TR-MOKE, thermal measurements and thermal modelling at the University of Illinois was supported by the US Department of Energy Office of Basic 
Energy Sciences under grant number DE-FG02-07ER46459. Measurements of thermally driven spin transport model were supported by the Army Research Office under grant number W911NF-11-10526. TR-MOKE and TDTR were carried out in the Laser and Spectroscopy Laboratory of the Materials Research Laboratory at the University of Illinois Urbana-Champaign. B.C.M. was supported by the Korea Institute of Science and Technology (KIST) institutional programme, the Pioneer Research Center Program of MSIP/NRF (2011-0027905) and the IT R\&D programme of MOTIE/KEIT (10043398). B.C.M. acknowledges the technical assistance from Jung-Min Han at KIST. K.J.L. was supported by the NRF (NRF-2013R1A2A2A01013188) and KU-KIST School Joint Research Program. K.J.L. acknowledges M.D. Stiles for discussion.

\section{Author contributions}

G.-M.C. designed the experiments together with B.-C.M. and D.G.C.; the samples were fabricated by G.-M.C. and B.-C.M.; the measurements were carried out by G.-M.C. with help of D.G.C.; the model calculation and data analysis were done by G.-M.C. and K.-J.L. together with D.G.C.; all authors discussed the results and wrote the manuscript.

\section{Additional information}

Supplementary Information accompanies this paper at http://www.nature.com/ naturecommunications

Competing financial interests: The authors declare no competing financial interests.

Reprints and permission information is available online at http://npg.nature.com/ reprintsandpermissions/

How to cite this article: Choi, G.-M. et al. Spin current generated by thermally driven ultrafast demagnetization. Nat. Commun. 5:4334 doi: 10.1038/ncomms5334 (2014). 